\title{
Efficacy of Biologicals in the Treatment of Rheumatoid Arthritis
}

\author{
A Meta-Analysis \\ S.P. Venkateshan S. Sidhu S. Malhotra P. Pandhi \\ Department of Pharmacology, Postgraduate Institute of Medical Education and Research, Chandigarh, India
}

\section{Key Words}

Rheumatoid arthritis $\cdot$ Therapies, new $\cdot$ Biologicals $\cdot$

Anti-tumour necrosis factor- $\alpha \cdot$ Anakinra $\cdot$ Abatacept

\begin{abstract}
Rheumatoid arthritis (RA) is a chronic multisystem disease. A characteristic feature of RA is persistent inflammatory synovitis, usually involving the peripheral joints in a symmetric distribution. The prevalence of RA is approximately $0.8 \%$ of the population (range: 0.3-2.1\%); women are affected approximately 3 times more often than men. The current therapeutic approach is to start a disease-modifying agent early in the illness to prevent eventual joint damage. Older disease-modifying anti-rheumatic drugs include methotrexate, sulphasalazine and hydroxychloroquine. Newer ones such as leflunomide and cyclosporin are also used. A recent advance in the management of rheumatoid arthritis is the use of biological agents, which block certain key molecules involved in the pathogenesis of the illness. They include tumour-necrosis-factor- $\alpha$-blocking agents such as infliximab, etanercept and adalimumab, the anti-CD-20 agent, rituximab, and CTLA-4 lg abatacept. The present study was planned with the aim of evaluating the efficacy of such newer biological therapies in refractory RA at various time points. Databases including Medline, Embase and the Cochrane Library were searched for all relevant studies up to January 2007. A total of 26 studies were included in present metaanalysis. The method of DerSimonian and Laird [Control Clin
\end{abstract}

Trials 1986;7:177-188] was used to calculated a pooled odds ratio (OR) for the American College of Rheumatology (ACR) criteria 20, 50 and 70, at 24, 54 and 96 weeks. The overall pooled OR were found to be significantly more than the placebo at all 3 time points for all 3 criteria (ACR 20, 50 70). In conclusion, biologicals as a group are highly effective in the treatment of RA. Biologicals were efficacious both in treatment naïve and methotrexate-refractory patients.

Copyright $\odot 2008$ S. Karger AG, Basel

\section{Introduction}

Rheumatoid arthritis (RA) is a chronic autoimmune disease, characterized by symmetrical synovitis, inflammatory exudates in joint cavities and erosion of articular cartilage and marginal bone [1]. Standard therapy for RA aims to suppress inflammation, a consequence of autoimmune activation, for which we use disease-modifying anti-rheumatic drugs (DMARD), corticosteroids and non-steroidal anti-inflammatory drugs [2]. However, a large number of patients treated with traditional DMARD showed an inadequate response [3]. This led to the development of new a class of drugs named biologicals [4].

Biologicals have highly specific action, targeting specific inflammatory proteins in the pathophysiology of RA. The most important of these are strategies targeting tumour necrosis factor- $\alpha$ (TNF- $\alpha)$; these are: TNF- $\alpha$

\section{KARGER}

Fax +4161306 1234

E-Mail karger@karger.ch

www.karger.com
(C) 2008 S. Karger AG, Base

0031-7012/09/0831-0001\$26.00/0

Accessible online at:

www.karger.com/pha
Dr. S.P. Venkateshan

Department of Pharmacology

Postgraduate Institute of Medical Education and Research

Research Block B, 4th Floor, Chandigarh (India)

Tel. +91 172275 5243, Fax +91 172274 4401, E-Mail venkydocs@yahoo.com 


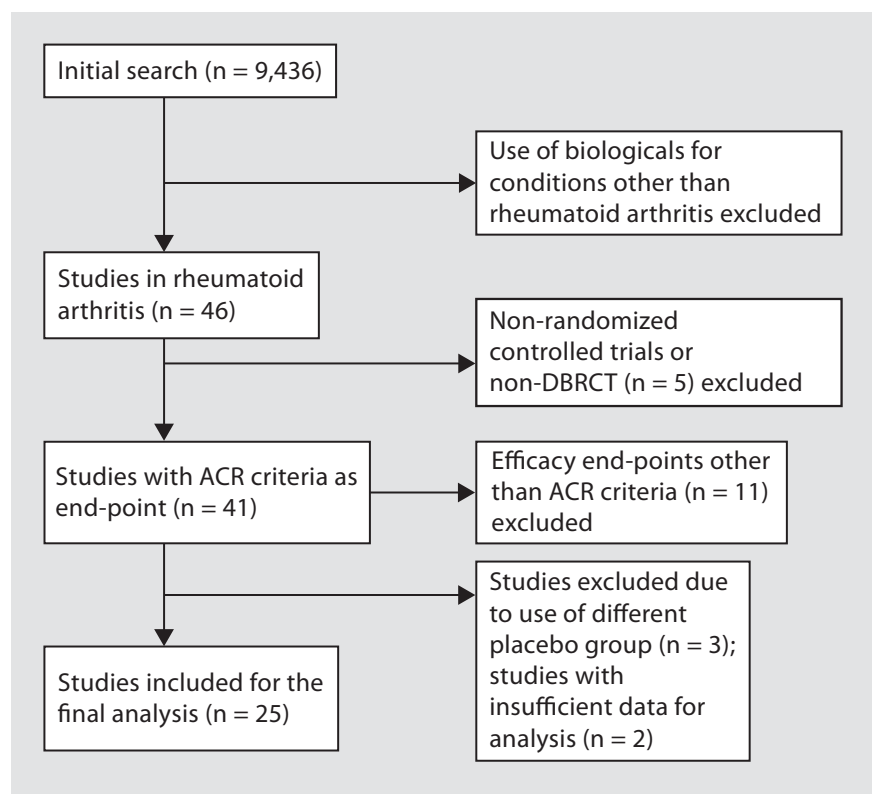

Fig. 1. Flow chart for studies evaluated for inclusion in the metaanalysis.

type II receptor/IgG1 fusion protein (etanercept), chimeric (human and mouse) monoclonal antibody against TNF- $\alpha$ (infliximab) and a humanized monoclonal antibody against TNF- $\alpha$ (adalimumab) $[5,6]$. Anakinra, a fusion protein that blocks actions of IL-1, has also been shown to be effective in clinical trials for RA [7]. Abatacept, a selective modulator of CD80 or CD86-CD28 costimulatory signals required for full $\mathrm{T}$ cell activation, is another agent recently shown to be effective. All these agents have been shown to be highly effective $[8,9]$. A number of other biologicals are being tested for efficacy in clinical trials for RA.

A large number of clinical trials have been conducted comparing biologicals with traditional DMARD [10]. The biologicals have shown superior efficacy, especially during the initial 6 months of treatment, but with progressive therapy this efficacy seems to decline, indicating disease progression. The results also vary with the type of biological used, in addition to a host of other patient characteristics [11]. Consequently, not all the trials have shown consistent improvement over the traditional DMARD at time intervals beyond 6 months. Lastly, as these newer therapies are expensive, the question of their continued use over a prolonged duration needs to be addressed.

The present meta-analysis was planned with the aim of comparing different biologicals to each other, tradi- tional DMARD and placebos, in terms of the American College of Rheumatology (ACR) criteria at different time points $(24,54$ and 96 weeks).

\section{Materials and Methods}

Two investigators independently searched databases, including Medline, Embase and the Cochrane Library, for all the relevant studies up to January 2007. The following terms were used: 'rheumatoid arthritis AND biologicals', 'anti-TNF- $\alpha$ AND rheumatoid arthritis', 'infliximab AND clinical trials', 'etanercept AND clinical trials', 'adalimumab AND clinical trials', and 'therapeutic uses AND biologicals'. Apart from the electronic search, Index Medicus and cross references of the articles obtained were also checked for any other useful data.

Studies included in the meta-analysis were randomized double-blind trials of infliximab, etanercept, adalimumab and abatacept, in which patients were randomized either to the biological or placebo group. Clinical trials meeting those criteria were collected. Studies were selected in which the patients had active RA (as defined by ACR criteria), despite treatment with traditional DMARD, and the treatment duration with biologicals was at least 12 weeks. For inclusion, the trials had to have a fixed dosage throughout the study period, without any dose titration of the biological. Studies were excluded if interventional studies were not randomized or double-blind. We compared all biological therapies (infliximab, etanercept, adalimumab, anakinra and abatacept) versus placebo alone, or all biological plus methotrexate (or DMARD) versus placebo plus methotrexate (or DMARD).

\section{Data Extraction}

Data regarding improvements in ACR 20, ACR 50 and ACR 70 criteria were extracted. Where an improvement in ACR criteria was represented as percentage of patients, it was converted into the number of patients. These improvements in ACR criteria were analyzed at time points of 24, 54 and 96 weeks after the start of treatment. The 24-week group included data from weeks 22 to 30 , the 54-week group from weeks 46 to 54 and the 96-week group from weeks 96 to 104 .

\section{Analysis}

For each trial, the odds ratio (OR) of the effects of biologicals versus placebo with $95 \%$ confidence intervals (CI) were calculated for ACR 20, 50 and 70 criteria. The random effects method of DerSimonian and Laird [12] was used to pool the data. The OR at different time points for ACR 20, 50 and 70 was plotted using forest plots. The OR was represented as a box with the 95\% CI as whiskers on both sides of the box. The size of each box in a forest plot was adjusted depending upon the weight of the study.

\section{Results}

In the initial search, 9,436 studies were identified (fig. 1), which included 246 meta-analyses and 654 reviews. A large number of clinical trials of biologicals in 
Table 1. Characteristics of studies included in the meta-analysis

\begin{tabular}{|c|c|c|c|c|c|c|}
\hline No. & Authors & $\begin{array}{l}\text { Subjects } \\
\text { random- } \\
\text { ized, } \mathrm{n}\end{array}$ & $\begin{array}{l}\text { Disease } \\
\text { charac- } \\
\text { teristics }\end{array}$ & Active treatment & Placebo & $\begin{array}{l}\text { Dura- } \\
\text { tion } \\
\text { weeks }\end{array}$ \\
\hline 1 & $\begin{array}{l}\text { Moreland } \\
\text { et al. [28] }\end{array}$ & 158 & active RA & etanercept 25 mg (78) & placebo $(80)$ & 24 \\
\hline 2 & $\begin{array}{l}\text { Weinblatt } \\
\text { et al. [29] }\end{array}$ & 89 & active RA & etanercept 25 mg + methotrexate (59) & placebo + methotrexate (30) & 24 \\
\hline 3 & $\begin{array}{l}\text { Klareskog } \\
\text { et al. [30] }\end{array}$ & 161 & active RA & etanercept 25 mg + methotrexate (85) & placebo + methotrexate (76) & 52 \\
\hline 4 & $\begin{array}{l}\text { Genovese } \\
\text { et al. [42] }\end{array}$ & 424 & active RA & etanercept $25 \mathrm{mg}+$ methotrexate (207) & placebo + methotrexate $(217)$ & 96 \\
\hline 5 & $\begin{array}{l}\text { Van der Heijde } \\
\text { et al. [31] }\end{array}$ & 146 & active RA & etanercept $25 \mathrm{mg}+$ methotrexate $(71)$ & placebo + methotrexate (75) & 96 \\
\hline 6 & $\begin{array}{l}\text { Maini } \\
\text { et al. [17] }\end{array}$ & 428 & active RA & $\begin{array}{l}\text { infliximab } 3 \mathrm{mg} \text { every } 4 \text { weeks }(86) \\
\text { infliximab } 3 \mathrm{mg} \text { every } 8 \text { weeks }(86) \\
\text { infliximab } 10 \mathrm{mg} \text { every } 4 \text { weeks }(81) \\
\text { infliximab } 10 \mathrm{mg} \text { every } 8 \text { weeks }(87)\end{array}$ & placebo (88) & 30 \\
\hline 7 & $\begin{array}{l}\text { Lipsky } \\
\text { et al. [5] }\end{array}$ & 428 & active RA & $\begin{array}{l}\text { infliximab } 3 \mathrm{mg} \text { every } 4 \text { weeks + methotrexate }(88) \\
\text { infliximab } 3 \mathrm{mg} \text { every } 8 \text { weeks }+ \text { methotrexate }(86) \\
\text { infliximab } 10 \mathrm{mg} \text { every } 4 \text { weeks }+ \text { methotrexate }(87) \\
\text { infliximab } 10 \mathrm{mg} \text { every } 8 \text { weeks + methotrexate }(81)\end{array}$ & placebo + methotrexate $(86)$ & 54 \\
\hline 8 & $\begin{array}{l}\text { Maini } \\
\text { et al. [18] }\end{array}$ & 428 & active RA & $\begin{array}{l}\text { infliximab } 3 \text { mg every } 4 \text { weeks (86) } \\
\text { infliximab } 3 \text { mg every } 8 \text { weeks (86) } \\
\text { infliximab } 10 \text { mg every } 4 \text { weeks }(81) \\
\text { infliximab } 10 \text { mg every } 8 \text { weeks }(87)\end{array}$ & placebo $(88)$ & 102 \\
\hline 9 & $\begin{array}{l}\text { St Clair } \\
\text { et al. [19] }\end{array}$ & 1,049 & active RA & $\begin{array}{l}\text { infliximab } 3 \mathrm{mg} \text { every } 8 \text { weeks + methotrexate }(372) \\
\text { infliximab } 6 \text { mg every } 8 \text { weeks + methotrexate }(377)\end{array}$ & placebo + methotrexate (291) & 54 \\
\hline 10 & $\begin{array}{l}\text { Quinn } \\
\text { et al. [20] }\end{array}$ & 20 & active RA & infliximab 3 mg (10) & placebo (10) & 46 \\
\hline 11 & $\begin{array}{l}\text { Smolen } \\
\text { et al. [21] }\end{array}$ & 428 & active RA & $\begin{array}{l}\text { infliximab } 3 \mathrm{mg} \text { every } 4 \text { weeks + methotrexate }(86) \\
\text { infliximab } 3 \mathrm{mg} \text { every } 8 \text { weeks }+ \text { methotrexate }(86) \\
\text { infliximab } 10 \mathrm{mg} \text { every } 4 \text { weeks + methotrexate }(81) \\
\text { infliximab } 10 \mathrm{mg} \text { every } 8 \text { weeks + methotrexate }(87)\end{array}$ & placebo + methotrexate (88) & 54 \\
\hline 12 & $\begin{array}{l}\text { Westhovens } \\
\text { et al. [22] }\end{array}$ & 1,082 & active RA & $\begin{array}{l}\text { infliximab } 3 \mathrm{mg} \text { at weeks } 0,2,6,14+\text { methotrexate } \\
(360) \\
\text { infliximab } 10 \mathrm{mg} \text { at weeks } 0,2,6,14+\text { methotrexate } \\
(361)\end{array}$ & placebo + methotrexate $(361)$ & 22 \\
\hline 13 & Rau [23] & 271 & active RA & $\begin{array}{l}\text { adalimumab } 20 \mathrm{mg} \text { every } 2 \text { weeks + methotrexate (70) } \\
\text { adalimumab } 40 \mathrm{mg} \text { every } 2 \text { weeks + methotrexate (70) } \\
\text { adalimumab } 80 \mathrm{mg} \text { every } 2 \text { weeks + methotrexate (70) }\end{array}$ & placebo + methotrexate $(70)$ & 24 \\
\hline 14 & $\begin{array}{l}\text { Furst } \\
\text { et al. [24] }\end{array}$ & 636 & active RA & adalimumab 40 mg every 2 weeks + DMARD (312) & placebo + DMARD (318) & 24 \\
\hline 15 & $\begin{array}{l}\text { Weinblatt } \\
\text { et al. [6] }\end{array}$ & 271 & active RA & $\begin{array}{l}\text { adalimumab } 20 \mathrm{mg} \text { every } 2 \text { weeks + methotrexate (69) } \\
\text { adalimumab } 40 \mathrm{mg} \text { every } 2 \text { weeks + methotrexate (67) } \\
\text { adalimumab } 80 \mathrm{mg} \text { every } 2 \text { weeks + methotrexate (73) }\end{array}$ & placebo + methotrexate $(62)$ & 24 \\
\hline 16 & $\begin{array}{l}\text { Keystone } \\
\text { et al. [25] }\end{array}$ & 618 & active RA & $\begin{array}{l}\text { adalimumab } 20 \mathrm{mg} / \text { week + methotrexate (212) } \\
\text { adalimumab } 40 \mathrm{mg} / \text { week + methotrexate (207) }\end{array}$ & placebo + methotrexate $(200)$ & 52 \\
\hline 17 & $\begin{array}{l}\text { Van de Putte } \\
\text { et al. [26] }\end{array}$ & 544 & active RA & $\begin{array}{l}\text { adalimumab } 20 \mathrm{mg} / \text { week (112) } \\
\text { adalimumab } 20 \mathrm{mg} \text { every } 2 \text { weeks (106) } \\
\text { adalimumab } 40 \mathrm{mg} / \text { week (103) } \\
\text { adalimumab } 40 \mathrm{mg} \text { every } 2 \text { weeks (113) }\end{array}$ & placebo (110) & 26 \\
\hline
\end{tabular}


Table 1 (continued)

\begin{tabular}{|c|c|c|c|c|c|c|}
\hline No. & Authors & $\begin{array}{l}\text { Subjects } \\
\text { random- } \\
\text { ized, n }\end{array}$ & $\begin{array}{l}\text { Disease } \\
\text { charac- } \\
\text { teristics }\end{array}$ & Active treatment & Placebo & $\begin{array}{l}\text { Dura- } \\
\text { tion } \\
\text { weeks }\end{array}$ \\
\hline 18 & $\begin{array}{l}\text { Breedveld } \\
\text { et al. [27] }\end{array}$ & 525 & active RA & $\begin{array}{l}\text { adalimumab } 40 \mathrm{mg} \text { every } 2 \text { weeks } \\
+ \text { methotrexate (268) }\end{array}$ & placebo + methotrexate $(257)$ & 104 \\
\hline 19 & $\begin{array}{l}\text { Kremer } \\
\text { et al. [3] }\end{array}$ & 339 & active RA & $\begin{array}{l}\text { CTLA-4 Ig } 2 \mathrm{mg} / \mathrm{kg}+\text { DMARD (105) } \\
\text { CTLA-4 Ig } 10 \mathrm{mg} / \mathrm{kg} \text { + DMARD (115) }\end{array}$ & placebo + DMARD (119) & 26 \\
\hline 20 & $\begin{array}{l}\text { Genovese } \\
\text { et al. [32] }\end{array}$ & 391 & active RA & abatacept 10 mg/kg + DMARD (258) & placebo + DMARD (133) & 26 \\
\hline 21 & $\begin{array}{l}\text { Kremer } \\
\text { et al. [34] }\end{array}$ & 339 & active RA & $\begin{array}{l}\text { CTLA-4 Ig } 2 \mathrm{mg} / \mathrm{kg}+\text { DMARD (105) } \\
\text { CTLA-4 Ig } 10 \mathrm{mg} / \mathrm{kg} \text { + DMARD (115) }\end{array}$ & placebo + DMARD (119) & 48 \\
\hline 22 & $\begin{array}{l}\text { Kremer } \\
\text { et al. [33] }\end{array}$ & 652 & active RA & abatacept $10 \mathrm{mg} / \mathrm{kg}$ + DMARD (433) & placebo + DMARD (219) & 48 \\
\hline 23 & $\begin{array}{l}\text { Bresnihan } \\
\text { et al. [35] }\end{array}$ & 349 & active RA & $\begin{array}{l}\text { anakinra } 75 \mathrm{mg}(115) \\
\text { anakinra } 150 \mathrm{mg}(115)\end{array}$ & placebo (119) & 24 \\
\hline 24 & $\begin{array}{l}\text { Cohen } \\
\text { et al. [36] }\end{array}$ & 153 & active RA & $\begin{array}{l}\text { anakinra } 1 \mathrm{mg} / \mathrm{kg}+\text { methotrexate (59) } \\
\text { anakinra } 2 \mathrm{mg} / \mathrm{kg} \text { + methotrexate (46) }\end{array}$ & placebo + methotrexate $(48)$ & 24 \\
\hline 25 & $\begin{array}{l}\text { Cohen } \\
\text { et al. [37] }\end{array}$ & 501 & active RA & anakinra $100 \mathrm{mg}+$ methotrexate $(250)$ & placebo + methotrexate $(251)$ & 24 \\
\hline
\end{tabular}

Figures in parentheses represent the number of participants.

conditions other than RA, herbal therapies for RA and observational studies were also included in the initial search. Forty-one potentially relevant studies were obtained. Of these, 11 were excluded on the basis of study end-points other than ACR criteria. Three studies were excluded due to the use of an active comparator other than methotrexate [13-15], and 2 were excluded as only 12 weeks of data were presented $[16,41]$.

Finally, studies evaluating 11,252 patients were included in the meta-analysis. Seven trials were of infliximab [5, 17-22], 6 evaluated adalimumab [6, 23-27], 5 studied etanercept [28-31,42], 4 investigated abatacept [3, 32-34], and 3 used anakinra [35-37] (tables 1,2).

In 3 studies, infliximab infusions were compared with the placebo and all patients had active disease, having received oral or parenteral methotrexate for at least 3 months and a stable dose of at least $12.5 \mathrm{mg} /$ week for at least 4 weeks [18, 19, 21]. In the other 4 studies, all the included patients received methotrexate in a stable dose around $20 \mathrm{mg} /$ week $[5,16,17,20]$. In 1 study, the methotrexate dose was $12.5 \mathrm{mg} / \mathrm{kg}$ [5].

One study compared etanercept directly with a placebo [29]. However, the other 4 studies used methotrexate in both the groups $[28,30-32]$. In study by Weinblatt et al. [29], the methotrexate dose was titrated to the desired clinical benefit before starting the etanercept infusions. However, in other studies, methotrexate was used orally in doses up to $20 \mathrm{mg} /$ week $[28,29,31,32]$.

One study, evaluating adalimumab, compared it directly with the placebo [27]. However, all other studies used methotrexate in both the groups after titrating to the fixed entry dose that was previously taken by the patients [6, 22-26].

Abatacept-treated patients, in the trail of Genovese et al. [32], were treatment resistant to TNF antagonists. In the trial, abatacept was compared to a placebo, but all the patients received at least 1 DMARD. In a study by Kremer et al. [33], patients received methotrexate (15 mg/week), although methotrexate at $10 \mathrm{mg} /$ week was acceptable if the patient had a history of toxicity. The duration of this study was 1 year; during the initial 6 months no dose adjustments were made for methotrexate, but after 6 months dose adjustments were allowed. In another study by Kremer et al. [34], 2 doses of abatacept were compared with a placebo in patients on methotrexate. In this study, methotrexate was used in doses ranging from 10 to 30 $\mathrm{mg} /$ week. All 3 studies evaluating anakinra used methotrexate up to $20 \mathrm{mg} /$ week in both the groups. 


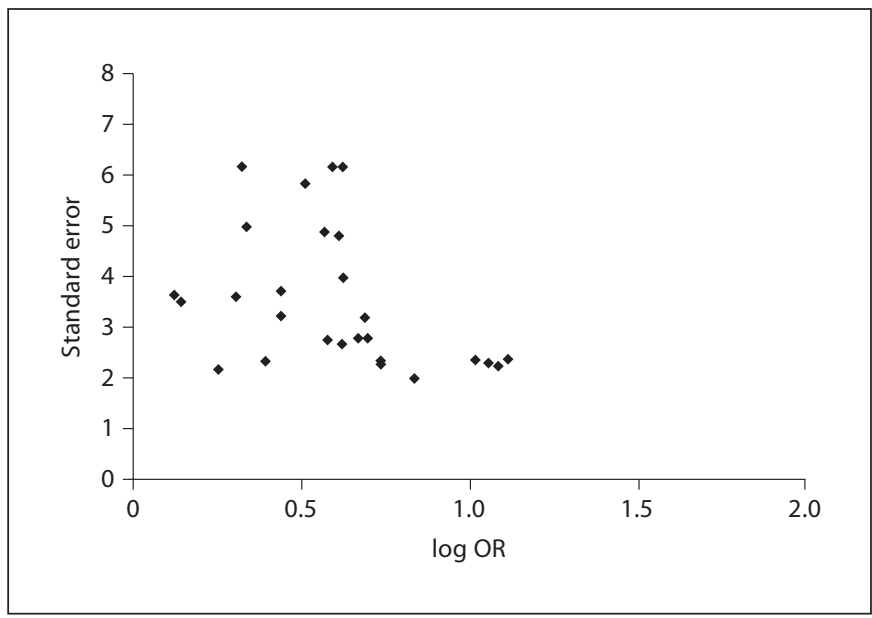

Fig. 2. Funnel plot of studies comparing biologicals with placebo.

Heterogeneity Test

From the shape of the funnel plot, publication bias cannot be ruled out (fig. 2).

\section{ACR 20 Improvement}

For the efficacy of biologicals at 24 weeks, 26 studies were analyzed. Biologicals showed better efficacy than the placebo, with OR $=3.69$ (95\% CI: 3.48-3.87; fig. 3a). Similarly, improvements in ACR 20 criteria at weeks 54 (16 studies) and 96 (8 studies) were significantly larger in the biological groups compared to the placebo group, with $\mathrm{OR}=3.31$ (95\% CI: 2.98-3.64) and OR $=3.0$ (95\% CI: 2.64-3.35), respectively (fig. 3a).

\section{ACR 50 Improvement}

As with ACR 20, the improvements in ACR 50 were better in biological groups compared to the placebo at all time points. The numbers of studies included in analysis were 24 (24-week group), 10 (54-week group) and 8 (96week group). The pooled OR were 4.26 (95\% CI: $4.06-$ 4.45) , 3.60 (95\% CI: 2.93-4.26) and 3.20 (95\% CI: $2.65-$ 3.76 ) at 24,54 and 96 weeks, respectively (fig. 3 b).

\section{ACR 70 Improvement}

The results of ACR 70 showed that biologicals were better than methotrexate at 2 time points: at week 24 (22 studies) the pooled $\mathrm{OR}=4.21$ (95\% CI: 3.92-4.50; fig. 3c), and at week 54 (4 studies) OR $=4.06$ (95\% CI: 2.45-5.67; fig. 3c). However, at week 96 (2 studies) the biologicals were not shown to be statistically better than placebo with $\mathrm{OR}=1.34$ (95\% CI: 0.01-2.69; fig. 3c).
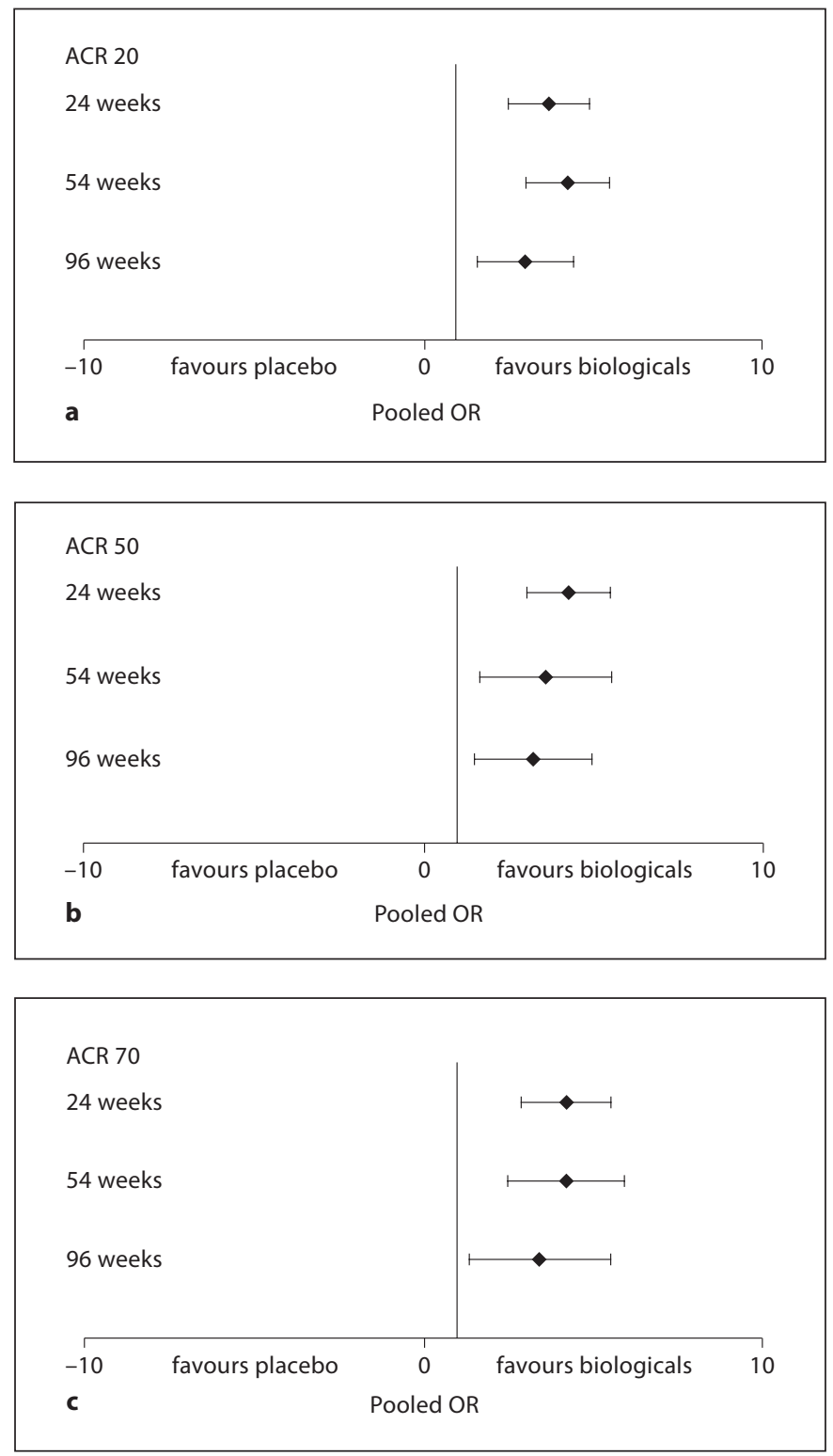

Fig. 3. Pooled OR for ACR 20 (a), 50 (b) and 70 (c) at 24, 54 and 96 weeks.

\section{Discussion}

Biologicals are currently indicated for the treatment of patients with active RA after an adequate trial of DMARD, most commonly methotrexate [34]. The results of the present meta-analysis suggest that in patients with an inadequate response to methotrexate, the addition of biologicals is beneficial, as defined by the ACR criteria. These findings further substantiate the available evidence in favour of biologicals.

Pharmacology 2009;83:1-9 
Table 2. Quality assessment of included studies

\begin{tabular}{|c|c|c|c|c|c|c|c|c|c|c|c|c|c|}
\hline \multirow[t]{2}{*}{ Checklist items } & \multirow{2}{*}{$\begin{array}{l}\text { Truly } \\
\text { ran- } \\
\text { dom }\end{array}$} & \multicolumn{2}{|c|}{ Randomization } & \multicolumn{3}{|c|}{ Baseline comparatibility } & \multicolumn{4}{|c|}{ Blinding } & \multicolumn{3}{|c|}{ Withdrawal } \\
\hline & & $\begin{array}{l}\text { allocation } \\
\text { conceal- } \\
\text { ment }\end{array}$ & $\begin{array}{l}\text { No. } \\
\text { stated }\end{array}$ & $\begin{array}{l}\text { pre- } \\
\text { sented }\end{array}$ & $\begin{array}{r}\text { achieved eligibility } \\
\text { criteria } \\
\text { specified }\end{array}$ & $\begin{array}{l}\text { co-inter- } \\
\text { ventions } \\
\text { identified }\end{array}$ & $\begin{array}{l}\text { asses- } \\
\text { sors }\end{array}$ & $\begin{array}{l}\text { admin- } \\
\text { istra- } \\
\text { tion }\end{array}$ & $\begin{array}{l}\text { partici- } \\
\text { pants }\end{array}$ & $\begin{array}{l}\text { proce- } \\
\text { dures } \\
\text { assessed }\end{array}$ & $\begin{array}{l}>80 \% \\
\text { in final } \\
\text { analysis }\end{array}$ & $\begin{array}{l}\text { rea- } \\
\text { sons } \\
\text { stated }\end{array}$ & $\begin{array}{l}\text { intention } \\
\text { to treat } \\
\text { analysis }\end{array}$ \\
\hline & 1 & 2 & 3 & 4 & 5 & 7 & 8 & 9 & 10 & 11 & 12 & 13 & 14 \\
\hline Moreland et al. [28] & & n.m & & & $\sqrt{ }$ & $\sqrt{ }$ & $\sqrt{ }$ & l & $\sqrt{ }$ & n.m & $\mathrm{x}$ & $\mathrm{v}$ & $\sqrt{ }$ \\
\hline Weinblatt et al. [29] & $\sqrt{ }$ & n.m & $\sqrt{ }$ & $\sqrt{ }$ & $\sqrt{ }$ & $\sqrt{ }$ & $\sqrt{ }$ & $\sqrt{ }$ & $\sqrt{ }$ & n.m & $\mathrm{x}$ & $\sqrt{ }$ & $\sqrt{ }$ \\
\hline Klareskog et al. [30] & $\sqrt{ }$ & n.m & 1 & 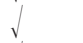 & $\sqrt{ }$ & $\sqrt{ }$ & $\sqrt{ }$ & $\sqrt{ }$ & $\sqrt{ }$ & n.m & $\mathrm{x}$ & $\sqrt{ }$ & $\sqrt{ }$ \\
\hline Genovese et al. [42] & 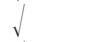 & n.m & $\sqrt{ }$ & $\sqrt{ }$ & $\sqrt{ }$ & $\sqrt{ }$ & $\sqrt{ }$ & $\sqrt{ }$ & $\sqrt{ }$ & n.m & $\mathrm{x}$ & $\sqrt{ }$ & $\sqrt{ }$ \\
\hline Van der Heijde et al. [31] & $\sqrt{ }$ & n.m & $\sqrt{ }$ & 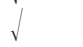 & $\sqrt{ }$ & $\sqrt{ }$ & $\sqrt{ }$ & $\sqrt{ }$ & $\sqrt{ }$ & n.m & $\mathrm{x}$ & $\sqrt{ }$ & $\sqrt{ }$ \\
\hline Maini et al. [17] & & n.m & 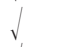 & & $\sqrt{ }$ & $\sqrt{ }$ & $\sqrt{ }$ & $\sqrt{ }$ & $\sqrt{ }$ & n.m & $\mathrm{x}$ & $\sqrt{ }$ & $\sqrt{ }$ \\
\hline Lipsky et al. [5] & $\mathrm{v}$ & n.m & $\sqrt{ }$ & r & $\sqrt{ }$ & $\sqrt{ }$ & $\sqrt{ }$ & $\sqrt{ }$ & $\sqrt{ }$ & n.m & $\mathrm{x}$ & $\sqrt{ }$ & $\sqrt{ }$ \\
\hline Maini et al. [18] & $\sqrt{ }$ & n.m & $\sqrt{ }$ & r & $\sqrt{ }$ & $\sqrt{ }$ & $\sqrt{ }$ & $\sqrt{ }$ & $\sqrt{ }$ & n.m & $\mathrm{x}$ & $\sqrt{ }$ & $\sqrt{ }$ \\
\hline St Clair et al. [19] & $\sqrt{ }$ & n.m & $\sqrt{ }$ & $v$ & $\sqrt{ }$ & $\sqrt{ }$ & $\sqrt{ }$ & $\sqrt{ }$ & $\sqrt{ }$ & n.m & $\mathrm{x}$ & & $\sqrt{ }$ \\
\hline Quinn et al. [20] & & n.m & $\sqrt{ }$ & $\sqrt{ }$ & $\sqrt{ }$ & $\sqrt{ }$ & $\sqrt{ }$ & $\sqrt{ }$ & $\sqrt{ }$ & n.m & $\mathrm{x}$ & $\sqrt{ }$ & $\sqrt{ }$ \\
\hline Smolen et al. [21] & & n.m & $\sqrt{ }$ & 1 & $\sqrt{ }$ & $\sqrt{ }$ & $\sqrt{ }$ & $\sqrt{ }$ & $\sqrt{ }$ & n.m & $\mathrm{x}$ & $\sqrt{ }$ & $\sqrt{ }$ \\
\hline Westhovens et al. [22] & $\sqrt{ }$ & n.m & $\sqrt{ }$ & $\sqrt{ }$ & $\sqrt{ }$ & $\sqrt{ }$ & $\sqrt{ }$ & $\sqrt{ }$ & $\sqrt{ }$ & n.m & $\mathrm{x}$ & $\sqrt{ }$ & $\sqrt{ }$ \\
\hline Rau [23] & $\sqrt{ }$ & n.m & $\sqrt{ }$ & $\sqrt{ }$ & $\sqrt{ }$ & $\sqrt{ }$ & $\sqrt{ }$ & $\sqrt{ }$ & $\sqrt{ }$ & n.m & $\mathrm{x}$ & $\sqrt{ }$ & $\sqrt{ }$ \\
\hline Furst et al. [24] & 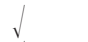 & n.m & $\sqrt{ }$ & $\sqrt{ }$ & $\sqrt{ }$ & $\sqrt{ }$ & $\sqrt{ }$ & $\sqrt{ }$ & $\sqrt{ }$ & n.m & $\mathrm{x}$ & $\sqrt{ }$ & $\sqrt{ }$ \\
\hline Weinblatt et al. [6] & $\sqrt{ }$ & n.m & $\sqrt{ }$ & $\sqrt{ }$ & $\sqrt{ }$ & $\sqrt{ }$ & $\sqrt{ }$ & $\sqrt{ }$ & $\sqrt{ }$ & n.m & $\mathrm{x}$ & $\sqrt{ }$ & $\sqrt{ }$ \\
\hline Keystone et al. [25] & $\sqrt{ }$ & n.m & $\sqrt{ }$ & $\sqrt{ }$ & $\sqrt{ }$ & $\sqrt{ }$ & $\sqrt{ }$ & $\sqrt{ }$ & $\sqrt{ }$ & n.m & $\mathrm{x}$ & $\sqrt{ }$ & $\sqrt{ }$ \\
\hline Van de Putte et al. [26] & $\sqrt{ }$ & n.m & $\sqrt{ }$ & $\sqrt{ }$ & $\sqrt{ }$ & $\sqrt{ }$ & $\sqrt{ }$ & $\sqrt{ }$ & $\sqrt{ }$ & n.m & $\mathrm{x}$ & $\sqrt{ }$ & $\sqrt{ }$ \\
\hline Breedveld et al. [27] & $\sqrt{ }$ & n.m & $\sqrt{ }$ & $\sqrt{ }$ & $\sqrt{ }$ & $\sqrt{ }$ & $\sqrt{ }$ & $\sqrt{ }$ & $\sqrt{ }$ & n.m & $\mathrm{x}$ & $\sqrt{ }$ & $\sqrt{ }$ \\
\hline Kremer et al. [3] & $\sqrt{ }$ & n.m & $\sqrt{ }$ & $\sqrt{ }$ & $\sqrt{ }$ & $\sqrt{ }$ & $\sqrt{ }$ & $\sqrt{ }$ & $\sqrt{ }$ & n.m & $\mathrm{x}$ & $\sqrt{ }$ & $\sqrt{ }$ \\
\hline Genovese et al. [32] & $\sqrt{ }$ & n.m & $\sqrt{ }$ & $\sqrt{ }$ & $\sqrt{ }$ & $\sqrt{ }$ & $\sqrt{ }$ & $\sqrt{ }$ & $\sqrt{ }$ & n.m & $\mathrm{x}$ & $\sqrt{ }$ & $\sqrt{ }$ \\
\hline Kremer et al. [34] & $\sqrt{ }$ & n.m & $\sqrt{ }$ & $\sqrt{ }$ & $\sqrt{ }$ & $\sqrt{ }$ & $\sqrt{ }$ & $\sqrt{ }$ & $\sqrt{ }$ & n.m & $\mathrm{x}$ & $\sqrt{ }$ & $\sqrt{ }$ \\
\hline Kremer et al. [33] & $\sqrt{ }$ & n.m & $\sqrt{ }$ & $\sqrt{ }$ & $\sqrt{ }$ & $\sqrt{ }$ & $\sqrt{ }$ & $\sqrt{ }$ & $\sqrt{ }$ & n.m & $\mathrm{x}$ & $\sqrt{ }$ & $\sqrt{ }$ \\
\hline Bresnihan et al. [35] & $\sqrt{ }$ & n.m & $\sqrt{ }$ & $\sqrt{ }$ & $\sqrt{ }$ & $\sqrt{ }$ & $\sqrt{ }$ & $\sqrt{ }$ & $\sqrt{ }$ & n.m & $\mathrm{x}$ & $\sqrt{ }$ & $\sqrt{ }$ \\
\hline Cohen et al. [36] & $\sqrt{ }$ & n.m & $\sqrt{ }$ & $\sqrt{ }$ & $\sqrt{ }$ & $\sqrt{ }$ & $\sqrt{ }$ & $\sqrt{ }$ & $\sqrt{ }$ & n.m & $\mathrm{x}$ & $\sqrt{ }$ & $\sqrt{ }$ \\
\hline Cohen et al. [37] & $\sqrt{ }$ & n.m & $\sqrt{ }$ & $\sqrt{ }$ & $\sqrt{ }$ & $\sqrt{ }$ & $\sqrt{ }$ & $\sqrt{ }$ & $\sqrt{ }$ & n.m & $\mathrm{x}$ & $\sqrt{ }$ & $\sqrt{ }$ \\
\hline
\end{tabular}

$\mathrm{n} . \mathrm{m}=$ Not mentioned .

Although overall biologicals were better than methotrexate in improving symptoms, individually some of the agents were not significantly better than methotrexate.

Week 24. The overall pooled OR favoured biologicals over methotrexate for ACR 20, 50 and 70. However, individually some studies were not significant. Anakinra, at all of the 5 doses analyzed, did not significantly improve ACR 20 when compared to methotrexate. A higher dose of abatacept (i.e. $10 \mathrm{mg} / \mathrm{kg}$ ) was more efficacious than a lower dose (i.e. $2 \mathrm{mg} / \mathrm{kg}$ ), and could achieve statistical significance. In the case of adalimumab, a weekly regimen was found to be better than a bi-weekly regimen, as statistically significant results were obtained with the weekly regimen. In the case of ACR 50, a study evaluating anakinra at doses of $1 \mathrm{mg} / \mathrm{kg}$ was the only study that attained significance; all the other doses did not attain statistical significance. Similarly to ACR 20, the lower dose of abatacept was not found to be significantly better than methotrexate. A lower dose of adalimumab (i.e. $20 \mathrm{mg} / \mathrm{kg}$ every week or every other week) did not significantly improve the ACR 50 response. Etanercept and anakinra did not significantly improve ACR 70. In the case of adalimumab, the study by Rau [23] did not show any significant improvement.

Week 54. Similar to results of week 24, at week 54 biologicals were better than methotrexate, in terms of overall pooled OR. Individually, the study that did not significantly favour biologicals for ACR 20 was study by Quinn et al. [20] using infliximab (3 mg/ $\mathrm{kg})$. The reason for the non-significant results obtained could the small sample size of the study, which was not sufficient to produce significant results. The other 2 studies that did not significantly improve ACR 20 were of adalimumab (biweekly regimen) and etanercept. ACR 50 studies using a lower dose of infliximab (i.e. $3 \mathrm{mg}$ ) were not significant, whereas higher doses (i.e. $10 \mathrm{mg}$ ) produced significant 
results. Individual OR for ACR 70 were not significant in cases where lower doses of infliximab and infliximab were given every 8 weeks. Adalimumab in a bi-weekly regimen did not significantly improve either ACR 50 or ACR 70 responses.

Week 96. At 96 weeks, Quinn et al [20] (infliximab) could not attain statistical significance in improving ACR 20,50 or 70 . Also, etanercept did not significantly improve any of the ACR criteria. Adalimumab in a biweekly regimen was also not significantly better than methotrexate at 96 weeks.

\section{Efficacy of Biologicals versus Duration of Treatment}

One of the most important issues regarding the use of biologicals for prolonged periods, taking into consideration the cost of the therapy, is maintained efficacy of treatment. Plotting OR for ACR 20, 50 and 70 versus time shows that biologicals are efficacious in terms of ACR criteria, but a uniform decline with time is observed (fig. 4).

\section{Methotrexate Dose}

Biologicals, when compared to placebo or methotrexate, have been shown to have better efficacy, but some of the trials have used low to moderate doses of methotrexate. The dose of methotrexate used in most of the studies was between 12 and $20 \mathrm{mg} /$ week. The starting dose being $12.5 \mathrm{mg} /$ week, and then titrated to around $20 \mathrm{mg} /$ week. These doses of methotrexate are considered to be low to moderate doses of methotrexate [39]. Doses between 15 and $25 \mathrm{mg} /$ week are considered to be appropriate. Additionally, in some of the trials, methotrexate was used in its oral form rather than though parenteral administration. This type of study design does not take into consideration the interindividual variations in the bioavailability of methotrexate. Usually, the bioavailability ranges between 25 and $100 \%$ [39]. Thus, oral methotrexate at 12-20 mg/week does not make a good comparator drug. Hence, based on results of prospective observational studies and known pharmacological properties of methotrexate, it has been suggested that any new drug should be compared with methotrexate given parenterally and in maximum weekly doses (i.e. up to $25 \mathrm{mg}$ ) [40].

\section{Dose of Biologicals Used}

In the trials included in the study, the dose of etanercept was $25 \mathrm{mg}$, given subcutaneously twice weekly, which is the standard FDA-approved dose, and most trials have evaluated this dose. Infliximab, however, has been used in doses ranging from 3 to $10 \mathrm{mg} / \mathrm{kg}$ given either every 4 weeks or every 8 weeks. Individual studies

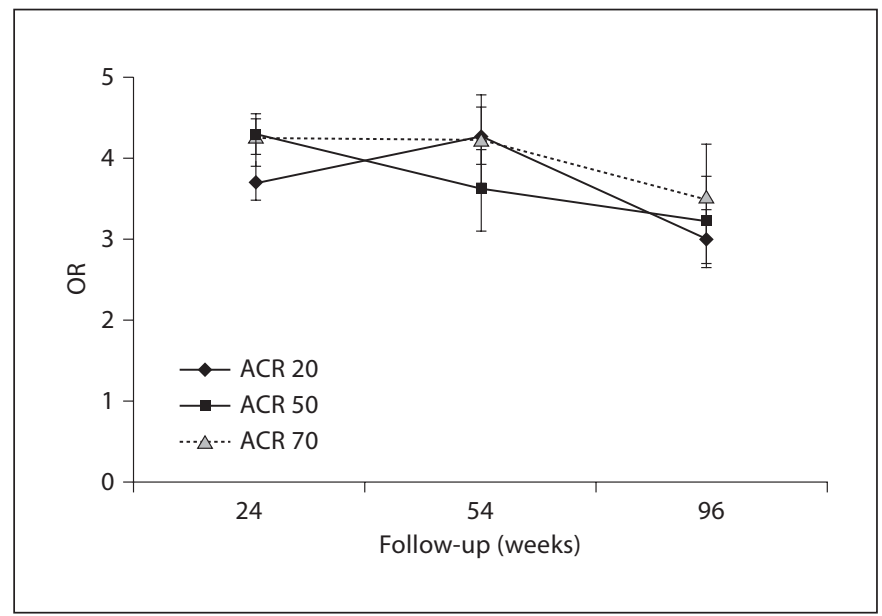

Fig. 4. Changes in ACR response rates for biologicals, measured as OR over time.

show a favourable response with higher doses of infliximab. In studies by Maini et al. $[17,18]$ and Lipsky et al. [5], higher or more frequent infliximab administration has shown better response rates. The higher dose needs to be titrated based on individual patient tolerability. Adalimumab is given either at 20,40 or $80 \mathrm{mg}$ every week/every other week. Again higher doses have shown better responses. As in case of infliximab, adalimumab administered every week was more efficacious than a biweekly regimen. Even in trials of anakinra, 5 different doses have been used, with higher response rates having higher doses. Abatacept, when used in a higher dose (i.e. $10 \mathrm{mg} / \mathrm{kg}$ ), was found to be better than a $2 \mathrm{mg} / \mathrm{kg}$ dose.

\section{Conclusion}

Biologicals as a group are highly effective in the treatment of RA. Biologicals were efficacious both in treatment naïve and methotrexate refractory patients. Individually, these agents have shown a trend towards improving the ACR response rate. Due consideration has to be given to the dose used in order to achieve an optimal response. Despite a decline in the response rate over time, biologicals are significantly better at all time points up to 96 weeks. In this meta-analysis, we analyzed 5 different biologicals acting on different protein targets. Combining these studies, biologicals, irrespective of the pathophysiological target they act on, are effective in the treatment of RA and improved the ACR 20,50 and 70 response rates at 24, 52 and 96 weeks. 


\section{References}

$\checkmark 1$ Lee DM, Weinblatt ME: Rheumatoid arthritis. Lancet 2001;358:903-911.

-2 American College of Rheumatology Subcommittee on Rheumatoid Arthritis Guidelines: Guidelines for management of rheumatoid arthritis: 2002 update. Arthritis Rheum 2002;46:328-346.

-3 Kremer JM, Westhovens R, Leon M, Eduardo Di Giorgio, Alten R, Steinfeld S, et al: Treatment of rheumatoid arthritis by selective inhibition of T-Cell activation with fusion protein CTLA4Ig. N Engl J Med 2003; 349:1907-1915.

4 Friestein GS: Evolving concepts of rheumatoid arthritis. Nature 2003;423:356-361.

5 Lipsky PE, van der Heijde DM, St Clair EW, Furst DE, Breedveld FC, Kalden JR: Infliximab and methotrexate in the treatment of rheumatoid arthritis: Anti-Tumor Necrosis Factor Trial in Rheumatoid Arthritis with Concomitant Therapy Study Group. N Engl J Med 2000;343:1594-1602.

-6 Weinblatt ME, Keystone EC, Furst DE, Moreland LW, Weisman MH, Birbara CA: Adalimumab, a fully human anti-tumor necrosis factor alpha monoclonal antibody, for the treatment of rheumatoid arthritis in patients taking concomitant methotrexate: the ARMADA trial. Arthritis Rheum 2003;48: 35-45.

7 Cohen SB: The use of anakinra, an interleukin-1 receptor antagonist, in the treatment of rheumatoid arthritis. Rheum Dis Clin North Am 2004;30:365-380.

-8 Moreland LW, Aethen R, Van den Bosch F, et al: Costimulatory blockade in patients with rheumatoid arthritis: a pilot, dose-finding study, double-blind, placebo-controlled clinical trial evaluating CTLA-4 Ig and LEA 29 Y eighty-five days after the first infusion. Arthritis Rheum 2002;46:1470-1479.

$\checkmark 9$ Emergy P: The therapeutic potential of costimulatory blockade with CTLA4 Ig in rheumatoid arthritis. Expert Opin Investig Drugs 2003;12:673-681.

10 Scott DL, Kingsley GH: Tumor necrosis factor inhibitors for rheumatoid arthritis. N Engl J Med 2006;355:704-712.

11 Genovese MC, Kremer JM: Treatment of rheumatoid arthritis with etanercept. Rheum Dis Clin North Am 2004;30:311328.

12 DerSimonian R, Larid N: Meta-analysis: a method for synthesizing research. Control Clin Trials 1986;7:177-188.

13 Durez P, Nzeusseu Toukap A, Lauwerys BR, Manicourt DH, Verschueren P, Westhovens $\mathrm{R}$, et al: A randomized comparative study of the short-term clinical and biological effects of intravenous pulse methylprednisolone and infliximab in patients with active rheumatoid arthritis despite methotrexate treatment. Ann Rheum Dis 2004;63:1069-1074.
14 Bathon JM, Martin RW, Fleischmann RM, Tesser JR, Schiff MH, Keystone EC, et al: A comparison of etanercept and methotrexate in patients with early rheumatoid arthritis. N Engl J Med 2000;343:1586-1593.

15 Johnsen AK, Schiff MH, Mease PJ, Moreland LW, Maier AL, Coblyn JS, et al: Comparison of 2 doses of etanercept (50 vs. $100 \mathrm{mg}$ ) in active rheumatoid arthritis: a randomized double blind study. J Rheumatol 2006;33: 659-664.

16 Lan JL, Chou SJ, Chen DY, Chen YH, Hsieh TY, Young M Jr: A comparative study of etanercept plus methotrexate and methotrexate alone in Taiwanese patients with active rheumatoid arthritis: a 12-week, double-blind, randomized, placebo-controlled study. J Formos Med Assoc 2004;103:618623.

17 Maini R, St Clair EW, Breedveld F, Furst D, Kalden J, Weisman M: Infliximab (chimeric anti-tumour necrosis factor alpha monoclonal antibody) versus placebo in rheumatoid arthritis patients receiving concomitant methotrexate: a randomized phase III trial. ATTRACT Study Group. Lancet 1999;354: 1932-1939.

18 Maini RN, Breedveld FC, Kalden JR, Smolen JS, Furst D, Weisman MH: Sustained improvement over two years in physical function, structural damage, and signs and symptoms among patients with rheumatoid arthritis treated with infliximab and methotrexate. Arthritis Rheum 2004;50:10511065.

19 St Clair EW, van der Heijde DM, Smolen JS, Maini RN, Bathon JM, Emery P: Combination of infliximab and methotrexate therapy for early rheumatoid arthritis: a randomized, controlled trial. Arthritis Rheum 2004; 50:3432-3443.

-20 Quinn MA, Conaghan PG, O'Connor PJ, Karim Z, Greenstein A, Brown A, et al: Very early treatment with infliximab in addition to methotrexate in early, poor-prognosis rheumatoid arthritis reduces magnetic resonance imaging evidence of synovitis and damage, with sustained benefit after infliximab withdrawal: results from a twelvemonth randomized, double-blind, placebocontrolled trial. Arthritis Rheum 2005;52:

$27-35$.
Smolen JS, Han C, Bala M, Maini RN, Kalden JR, van der Heijde D: Evidence of radiographic benefit of treatment with infliximab plus methotrexate in rheumatoid arthritis patients who had no clinical improvement: a detailed subanalysis of data from The AntiTumor Necrosis Factor Trial in Rheumatoid Arthritis with Concomitant Therapy Study. Arthritis Rheum 2005;52:1020-1030.
22 Westhovens R, Yocum D, Han J, Berman A, Strusberg I, Geusens P, Rahman MU; START Study Group: The safety of infliximab, combined with background treatments, among patients with rheumatoid arthritis and various comorbidities: a large, randomized, placebo-controlled trial (erratum in Arthritis Rheum 2007;56:1675). Arthritis Rheum 2006;54:1075-1086.

23 Rau R: Adalimumab (a fully human anti-tumour necrosis factor alpha monoclonal antibody) in the treatment of active rheumatoid arthritis: the initial results of five trials. Ann Rheum Dis 2002;61(suppl 2):ii70-ii73.

24 Furst DE, Schiff MH, Fleischmann RM, Strand V, Birbara CA, Compagnone D, Fischkoff SA, Chartash EK: Adalimumab, a fully human anti tumor necrosis factor-alpha monoclonal antibody, and concomitant standard antirheumatic therapy for the treatment of rheumatoid arthritis: results of STAR (Safety Trial of Adalimumab in Rheumatoid Arthritis). J Rheumatol 2003;30: 2563-2571.

25 Keystone EC, Kavanaugh AF, Sharp JT, Tannenbaum H, Hua Y, Teoh LS, Fischkoff SA, Chartash EK: Radiographic, clinical, and functional outcomes of treatment with adalimumab (a human anti-tumor necrosis factor monoclonal antibody) in patients with active rheumatoid arthritis receiving concomitant methotrexate therapy: a randomized, placebo-controlled, 52-week trial. Arthritis Rheum 2004;50:1400-1411.

-26 Van de Putte LB, Atkins C, Malaise M, et al: Efficacy and safety of adalimumab as monotherapy in patients with rheumatoid arthritis for whom previous disease modified antirheumatic drug treatment has failed. Ann Rheum Dis 2004;63:508-516.

27 Breedveld FC, Weisman MH, Kavanaugh AF, Cohen SB, Pavelka K, van Vollenhoven $\mathrm{R}$, et al: The PREMIER study: a multicenter, randomized, double-blind clinical trial of combination therapy with adalimumab plus methotrexate versus methotrexate alone or adalimumab alone in patients with early, aggressive rheumatoid arthritis who had not had previous methotrexate treatment. Arthritis Rheum 2006;54:26-37.

28 Moreland LW, Schiff MH, Baumgartner SW, Tindall EA, Fleischmann RM, Bulpitt KJ: Etanercept therapy in rheumatoid arthritis: a randomized, controlled trial. Ann Intern Med 1999;130:478-486.

29 Weinblatt ME, Kremer JM, Bankhurst AD, Bulpitt KJ, Fleischmann RM, Fox RI: A trial of etanercept, a recombinant tumor necrosis factor receptor: $\mathrm{Fc}$ fusion protein, in patients with rheumatoid arthritis receiving methotrexate. N Engl J Med 1999;340:253-259. 
>30 Klareskog L, van der Heijde D, de Jager JP, Gough A, Kalden J, Malaise M, et al: Therapeutic effect of the combination of etanercept and methotrexate compared with each treatment alone in patients with rheumatoid arthritis: double-blind randomised controlled trial. Lancet 2004;363:675-681.

-31 van der Heijde D, Klareskog L, Singh A, Tornero J, Melo-Gomes J, Codreanu C: Patient reported outcomes in a trial of combination therapy with etanercept and methotrexate for rheumatoid arthritis: the TEMPO trial. Ann Rheum Dis 2006;65:328-334.

-32 Genovese MC, Becker JC, Schiff M, Luggen M, Sherrer Y, Kremer J: Abatacept for rheumatoid arthritis refractory to tumor necrosis factor alpha inhibition. N Engl J Med 2005; 353:1114-1123.

>33 Kremer JM, Genant HK, Moreland LW, Russell AS, Emery P, Abud-Mendoza C, et al: Effects of abatacept in patients with methotrexate-resistant active rheumatoid arthritis. Ann Intern Med 2006;144:865-876.

-34 Kremer JM, Dougados M, Emery P, Durez P, Sibilia J, Shergy W, et al: Treatment of rheumatoid arthritis with the selective costimulation modulator abatacept. Arthritis Rheum 2005;52:2263-2271.
35 Bresnihan B, Alvaro-Gracia JM, Cobby M, et al: Treatment of rheumatoid arthritis with recombinant human interleukin-1 receptor antagonist. Arthritis Rheum 1998;41:21962204.

36 Cohen S, Hurd E, Cush J, et al: Treatment of rheumatoid arthritis with anakinra, a recombinant human interleukin-1 receptor antagonist with methotrexate: results of a twenty-four-week, multicenter, randomized, double-blind, placebo-controlled trial. Arthritis Rheum 2002;46:614-624.

37 Cohen S, Moreland LW, Cush J, et al: Anakinra (recombinant interleukin-1 receptor antagonist): a large placebo-controlled efficacy trial of anakinra in patients with erosive rheumatoid arthritis disease (abstract). In ACR/ARHP 65th Annu Sci Meet, San Francisco, 2001.

38 Furst DE, Breedveld FC, Kalden JR, Smolen JS, Antoni CE, Biljsma JWJ, et al: Updated consensus statement on biological agents for the treatment of rheumatoid arthritis and other rheumatic diseases (May 2002). Ann Rheum Dis 2002;61(suppl 2):ii2-ii7.
39 Seitz M, Villiger PM: Dosage and mode of administration of methotrexate in clinical trials: comment on the article by Genovese et al. Arthritis Rheum 2003;48:863.

$>40$ Oguey D, Kolliker F, Gerber NJ, et al: Effect of food on the bioavailability of low-dose methotrexate in patients with rheumatoid arthritis. Arthritis Rheum 1992;35:611-614.

41 van de Putte LB, Rau R, Breedveld FC, Kalden JR, Malaise MG, van Riel PL, Schattenkirchner M, Emery P, Burmester GR, Zeidler H, Moutsopoulos HM, Beck K, Kupper H: Efficacy and safety of the fully human antitumour necrosis factor alpha monoclonal antibody adalimumab (D2E7) in DMARD refractory patients with rheumatoid arthritis: a 12 week, phase II study. Ann Rheum Dis 2003;62:1168-1177.

42 Genovese MC, Bathon JM, Martin RW, Fleischmann RM, Tesser JR, Schiff MH, Keystone EC, Wasko MC, Moreland LW, Weaver AL, Markenson J, Cannon GW, Spencer-Green G, Finck BK: Etanercept versus methotrexate in patients with early rheumatoid arthritis: two-year radiographic and clinical outcomes. Arthritis Rheum 2002; 46:1443-1450. 